\title{
High-resolution molecular identification of smalltooth sawfish prey
}

\author{
Taylor L. Hancock ${ }^{1}$, Gregg R. Poulakis ${ }^{2}$, Rachel M. Scharer ${ }^{2}$, S. Gregory Tolley ${ }^{3}$ \& \\ Hidetoshi Urakawa ${ }^{1 *}$
}

The foundation of food web analysis is a solid understanding of predator-prey associations. Traditional dietary studies of fishes have been by stomach content analysis. However, these methods are not applicable to Critically Endangered species such as the smalltooth sawfish (Pristis pectinata). Previous research using the combination of stable isotope signatures from fin clips and 18S rRNA gene sequencing of fecal samples identified the smalltooth sawfish as piscivorous at low taxonomic resolution. Here, we present a high taxonomic resolution molecular technique for identification of prey using opportunistically acquired fecal samples. To assess potential biases, primer sets of two mitochondrial genes, 12S and 16S rRNA, were used alongside 18S rRNA, which targets a wider spectrum of taxa. In total, 19 fish taxa from 7 orders and 11 families native to the Gulf of Mexico were successfully identified. The sawfish prey comprised diverse taxa, indicating that this species is a generalist piscivore. These findings and the molecular approach used will aid recovery planning for the smalltooth sawfish and have the potential to reveal previously unknown predator-prey associations from a wide range of taxa, especially rare and hard to sample species.

The smalltooth sawfish (Pristis pectinata) currently inhabits southwest Florida and the Florida Keys but was once widely distributed on both coasts of the Atlantic Ocean and in the Gulf of Mexico ${ }^{1}$. Decades of human activity, including bycatch mortality in commercial and recreational fishing and loss of red mangrove (Rhizophora mangle) shorelines associated with residential and commercial development, have greatly reduced the size of the smalltooth sawfish population ${ }^{1,2}$. Thus, in 2003 the species was listed as endangered under the U.S. Endangered Species Act.

Despite recent expansion of knowledge about the smalltooth sawfish ${ }^{3}$, its feeding ecology is poorly understood. To maximize the effectiveness of ongoing recovery planning, more detailed knowledge of the trophic ecology of smalltooth sawfish is needed to better understand what specific prey they rely on. Traditional dietary studies on piscivores typically involve lethal sampling for stomach content analysis, gastric lavage, and morphological hard part analysis of indigestible prey remains ${ }^{4-7}$. Morphological characterization of prey remains found during gastric lavage or lethal sampling permits conclusions to be drawn about the number and size of fish prey consumed ${ }^{8,9}$; however, lethal sampling is not feasible for endangered species and gastric lavage, though considered non-invasive, may not be a permitted activity for endangered species ${ }^{10}$. Therefore, alternative approaches to identify predator-prey associations are warranted. Recent research using the combination of stable isotope signatures from fin clips and $18 \mathrm{~S}$ rRNA gene sequencing of fecal samples identified the smalltooth sawfish as piscivorous at low taxonomic resolution ${ }^{11}$. However, a higher taxonomic resolution understanding of this species' diet is needed to implement effective recovery planning through species-specific management of its prey base, which may include popular commercial and sport fishes.

Here we present a high-resolution molecular method for identification of smalltooth sawfish prey using fecal samples collected over a six-year period. To assess potential biases that might be caused by the selection of primer sets, two mitochondrial genes, $12 \mathrm{~S}$ and $16 \mathrm{~S}$ rRNA, were used together with $18 \mathrm{~S}$ rRNA, a more evolutionarily conserved gene that targets a wider spectrum of taxa ${ }^{11}$. Based on Poulakis and colleagues ${ }^{11}$ previous findings, we expected to find that smalltooth sawfish fed upon rays and various teleost fish species, some of which may be

\footnotetext{
${ }^{1}$ Department of Ecology and Environmental Studies, The Water School, Florida Gulf Coast University, Fort Myers, Florida, 33965, USA. 'Fish and Wildlife Research Institute, Florida Fish and Wildlife Conservation Commission, Charlotte Harbor Field Laboratory, Port Charlotte, Florida, 33954, USA. ${ }^{3}$ Department of Marine and Earth Sciences, The Water School, Florida Gulf Coast University, Fort Myers, Florida, 33965, USA. *email: hurakawa@fgcu.edu
} 


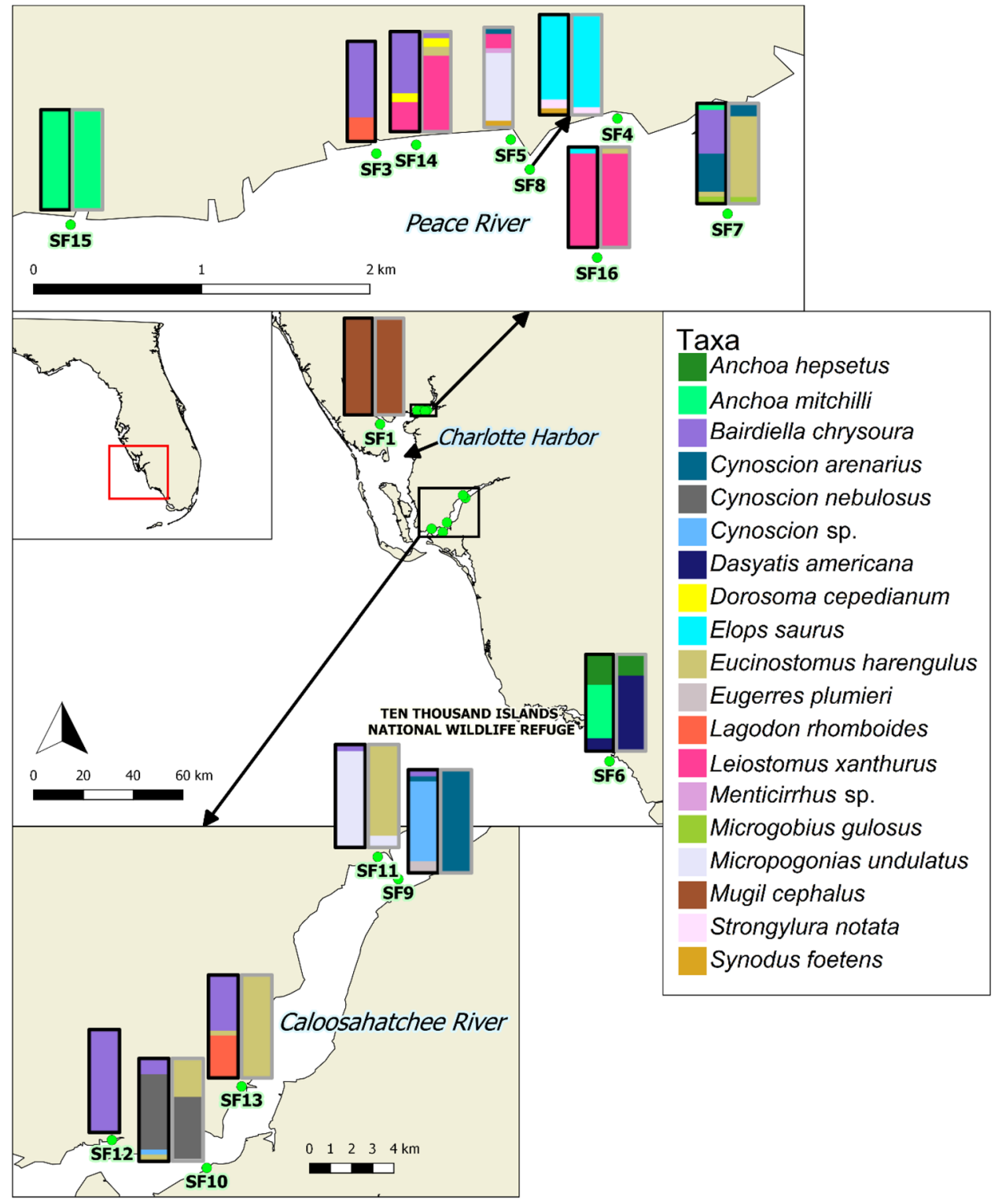

Figure 1. Map of smalltooth sawfish fecal sample collection locations in southwest Florida with stacked bars showing relative composition of fish prey taxa (minimum of 5\%), after removal of host sequences. Each $100 \%$ stacked bar shows data from mitochondrial 12S (black border) and 16S (grey border) rRNA genes. No fish prey taxa were detected in 16S rRNA gene analysis of samples SF3, SF4, and SF12. DNA sequencing of 12S rRNA gene failed in SF4 and SF5. SF2 did not yield enough DNA for analysis from its extraction. Map generated with QGIS Desktop 2.18 (https://www.qgis.org/en/site/) using data provided by the Florida Fish and Wildlife Conservation Commission-Fish and Wildlife Research Institute.

of anthropogenic interest, putting them in direct competition with humans. These data will improve recovery planning for this Critically Endangered species and the analysis technique has the potential to reveal previously unknown predator-prey associations of a wide range of taxa, especially rare and hard to sample species.

\section{Results}

Smalltooth sawfish fecal sample description. During field sampling from 2010 through 2015,16 fecal samples were opportunistically obtained from primarily juvenile $(<2500 \mathrm{~mm})$ smalltooth sawfish $(780-4355 \mathrm{~mm}$ stretch total length; mean $=1398 \mathrm{~mm})$ in a roughly equal proportion of males $(n=8)$ and females $(n=7)$ in southwest Florida from the Charlotte Harbor estuarine system and the Ten Thousand Islands National Wildlife Refuge (Fig. 1; Table 1). DNA was unable to be extracted from one sample due to an insufficient amount of feces.

18S rRNA gene analysis. The mean number of analyzed reads for each sample was $110,843 \pm 39,647$ ( \pm SE; Supplementary Table S1). After normalization (scaled to 10,000 reads) and subsequent removal of host sequences (i.e., smalltooth sawfish), four Kingdoms were identified: Animalia (91.3\%), Chromalveolata (6.2\%), 


\begin{tabular}{|l|l|l|l|l|l|}
\hline $\begin{array}{l}\text { Sample } \\
\text { code }^{*}\end{array}$ & Date & Location & Latitude, longitude & $\begin{array}{l}\text { STL** } \\
(\mathbf{m m})\end{array}$ & Sex \\
\hline SF1 $^{\dagger}$ & $7 / 31 / 2015$ & Upper Charlotte Harbor & $26.91680,-82.18010$ & 4355 & F \\
\hline SF3 & $7 / 13 / 2015$ & Peace River & $26.97085,-82.02990$ & 1020 & F \\
\hline SF4 & $4 / 9 / 2015$ & Peace River & $26.97272,-82.01702$ & 780 & M \\
\hline SF5 & $5 / 13 / 2014$ & Peace River & $26.97160,-82.02272$ & 825 & F \\
\hline SF6 & $4 / 19 / 2015$ & Ten Thousand Islands & $25.70193,-81.35288$ & 1690 & F \\
\hline SF7 & $5 / 28 / 2015$ & Peace River & $26.96660,-82.01118$ & 1020 & M \\
\hline SF8 & $6 / 12 / 2014$ & Peace River & $26.97000,-82.02170$ & 1556 & F \\
\hline SF9 & $4 / 23 / 2015$ & Caloosahatchee River & $26.65053,-81.87252$ & 1447 & M \\
\hline SF10 & $9 / 22 / 2015$ & Caloosahatchee River & $26.52737,-81.95422$ & 1235 & M \\
\hline SF11 & $5 / 22 / 2014$ & Caloosahatchee River & $26.66002,-81.88132$ & 958 & F \\
\hline SF12 & $12 / 21 / 2010$ & Caloosahatchee River & $26.53923,-81.99467$ & 2026 & M \\
\hline SF13 & $6 / 24 / 2010$ & Caloosahatchee River & $26.56210,-81.93932$ & 1025 & F \\
\hline SF14 & $9 / 3 / 2010$ & Peace River & $26.97132,-82.02777$ & 1380 & M \\
\hline SF15 & $5 / 1 / 2013$ & Peace River & $26.96653,-82.04620$ & 835 & M \\
\hline SF16 & $4 / 3 / 2011$ & Peace River & $26.96465,-82.01815$ & 810 & M \\
\hline
\end{tabular}

Table 1. Collection data associated with smalltooth sawfish fecal samples. *SF2 did not yield enough DNA for analysis from its extraction. ${ }^{* *} \mathrm{STL}=$ stretch (maximum) total length; mean $1398 \mathrm{~mm} \pm \mathrm{SE} 232 \mathrm{~mm}$. ${ }^{\dagger}$ Sample obtained from necropsy. ${ }^{\ddagger}$ Sample used in previous $18 \mathrm{~S}$ rRNA gene analysis (11).

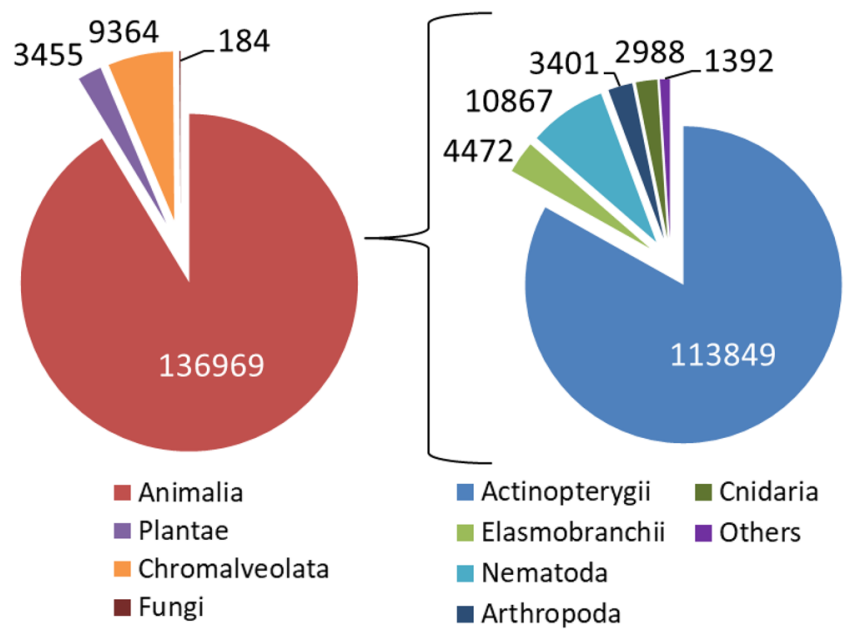

Figure 2. Abundance of normalized $18 \mathrm{~S}$ rRNA gene sequence reads by Kingdom (left) and composition of Animalia (right) from smalltooth sawfish fecal samples after removal of host sequences. Animalia composed 91.3\% of sequence reads (left), with fishes (Actinopterygii and Elasmobranchii) composing $86.4 \%$ of Animalia sequence reads (right). Others includes Mollusca and Platyhelminthes.

Plantae (2.4\%), and Fungi (0.1\%) (Fig. 2). All non-animal taxa were microscopic and considered to be of sediment or ambient water origin. Within Animalia, fishes comprised the majority of sequences (Actinopterygii: $83.1 \%$; Elasmobranchii: $3.3 \%$ ). In the majority of samples, Arthropoda sequences made up $<1 \%$ of Animalia sequences; however, considerable numbers were identified in one sample, SF4, with $99.2 \%$ of Animalia sequences and $100 \%$ of Arthropoda sequences identified as penaeid shrimp (Supplementary Table S2), with $91.5 \%$ of these sequences exhibiting 99\% similarity to reference sequences (Litopenaeus setiferus: JX403844.1; Farfantepenaeus duorarum: JX403828.1). SF4 was the smallest individual in our analysis $(780 \mathrm{~mm})$ and had no fish prey detected in our mitochondrial $16 \mathrm{~S}$ rRNA gene analysis (Fig. 3).

Mitochondrial 12S and 16S rRNA gene analysis. Preliminary results revealed large amounts of unidentified sequences present in our samples and a lack of local fish species reference sequences in GenBank, indicating the need for additional sequencing effort. Thus, we sequenced 24 fish species, including nine orders and 13 families, known to inhabit our sampling area ${ }^{12}$, yielding 16 and 23 additional sequences for the mitochondrial $12 S$ and 16S rRNA genes, respectively (Supplementary Table S3). The high specificity of these sequences allowed closely related species to be distinguished in most cases, which was beneficial in determining the highest resolution (i.e., species level) identifications for Operational Taxonomic Units (OTUs) with Basic Local Alignment Search Tool (BLAST) results that did not meet our identification criterion ( $98 \%$ similarity) or exhibited ties. To provide even more robust analysis, species signature sequences consisting of $\sim 40 \mathrm{bp}$ within $12 \mathrm{~S}$ and $16 \mathrm{~S}$ rRNA were identified 


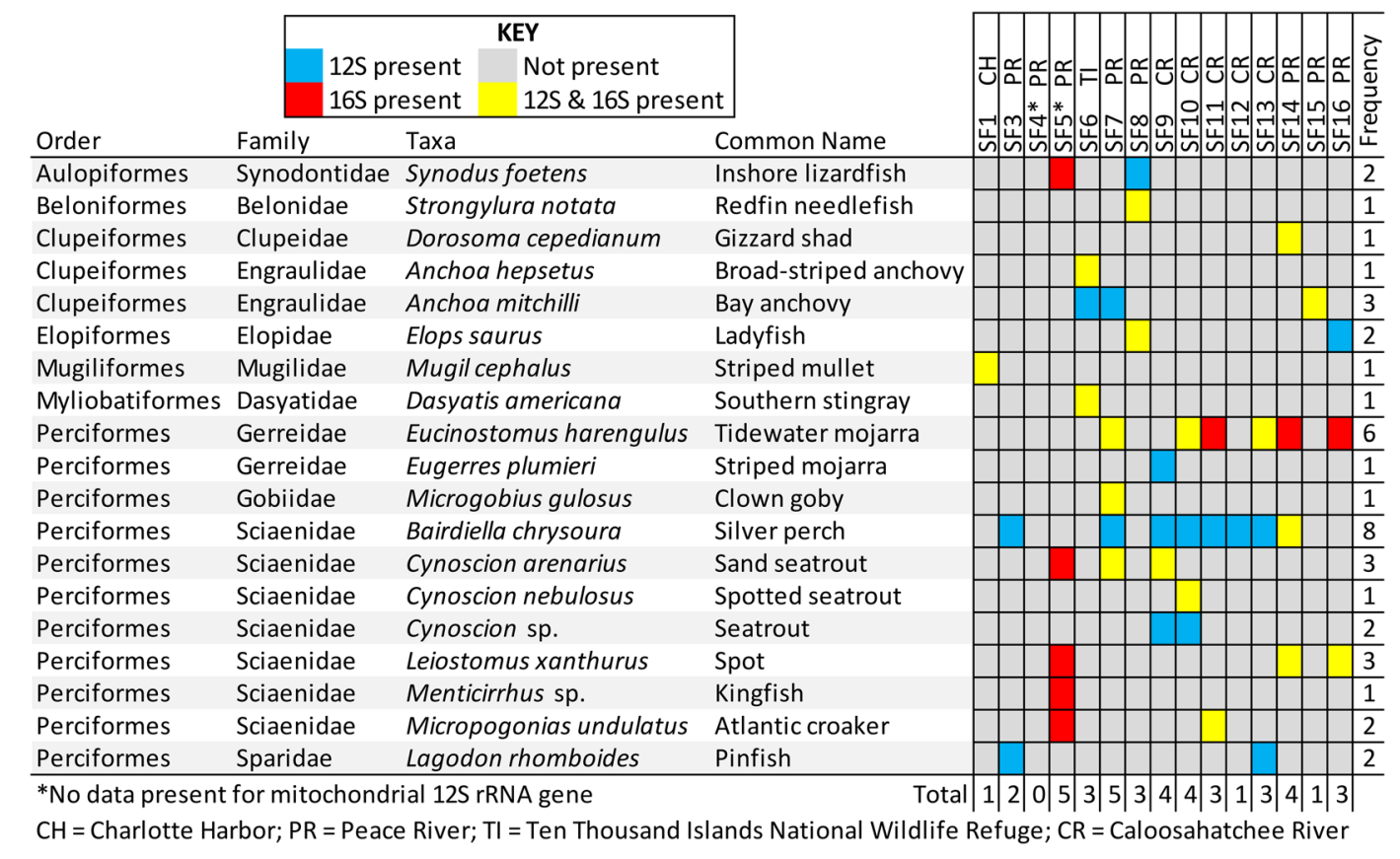

Figure 3. Comparison of fish prey taxa detection in smalltooth sawfish fecal samples using mitochondrial $12 \mathrm{~S}$ and 16S rRNA gene sequences. Sample locations indicated next to sample code (see footnote). SF2 did not yield enough DNA for analysis from its extraction.

\begin{tabular}{|c|c|c|c|c|c|c|}
\hline \multirow[b]{2}{*}{ Sample code } & \multicolumn{2}{|c|}{$\begin{array}{l}\text { Analyzed } \\
\text { sequence reads }\end{array}$} & \multicolumn{2}{|c|}{$\begin{array}{l}\text { Smalltooth } \\
\text { sawfish } \\
\text { sequences (\%) }\end{array}$} & \multicolumn{2}{|c|}{$\begin{array}{l}\text { Fish prey } \\
\text { sequence } \\
\text { identified } \\
(\%)^{\dagger}\end{array}$} \\
\hline & $12 S$ & $16 S$ & $12 S$ & $16 S$ & $12 S$ & $16 S$ \\
\hline SF1 & 5456 & 21202 & 82.3 & 99.9 & 91.0 & 75.0 \\
\hline SF3 & 2993 & 3026 & 11.3 & 100.0 & 95.4 & 0 \\
\hline SF4 & ND* & 16344 & ND & 100.0 & ND & 0 \\
\hline SF5 & ND & 14967 & ND & 31.4 & ND & 90.1 \\
\hline SF6 & 3675 & 24865 & 93.3 & 99.7 & 76.7 & 40.7 \\
\hline SF7 & 4199 & 11418 & 48.8 & 62.8 & 89.6 & 98.3 \\
\hline SF8 & 5958 & 12732 & 2.0 & 40.8 & 96.0 & 94.4 \\
\hline SF9 & 4783 & 8607 & 19.4 & 70.2 & 97.3 & 98.9 \\
\hline SF10 & 7300 & 9213 & 2.3 & 17.4 & 94.6 & 98.1 \\
\hline SF11 & 9702 & 7080 & 95.4 & 97.6 & 81.3 & 91.3 \\
\hline SF12 & 7371 & 8584 & 73.2 & 100.0 & 91.0 & 0 \\
\hline SF13 & 16437 & 10169 & 90.1 & 98.1 & 87.5 & 97.4 \\
\hline SF14 & 4945 & 9795 & 0.5 & 31.9 & 90.4 & 84.4 \\
\hline SF15 & 10896 & 4248 & 0.7 & 41.3 & 99.7 & 99.1 \\
\hline SF16 & 933 & 4069 & 5.3 & 24.1 & 97.1 & 99.1 \\
\hline Total & 84648 & 166319 & - & - & - & - \\
\hline Mean & 6511 & 11088 & 40.3 & 67.7 & 91.3 & 88.9 \\
\hline$\pm \mathrm{SE}$ & \pm 1112 & \pm 1593 & \pm 11.3 & \pm 8.6 & \pm 1.8 & \pm 4.9 \\
\hline
\end{tabular}

Table 2. Analyzed sequence reads of mitochondrial $12 \mathrm{~S}$ and $16 \mathrm{~S}$ rRNA genes and relative abundance of host and fish prey sequences. ${ }^{*} \mathrm{ND}=$ no data. ${ }^{\dagger}$ After removal of host sequences.

for 150 and 128 species (Supplementary Table S4), respectively, to conduct manual alignments of unidentified sequences during the final step of our identification protocol. This tag-sequencing strategy attributed to $17.4 \%$ and $2.8 \%$ of identified $12 \mathrm{~S}$ and $16 \mathrm{~S}$ rRNA sequences, respectively (Supplementary Table S5).

The mean $( \pm$ SE) number of analyzed reads for all samples was $8,963 \pm 1,074$. Two samples (SF4, SF5) failed to sequence for $12 \mathrm{~S}$ rRNA analysis due to unsuccessful PCR amplicon generation. After removal of host sequences, our analysis identified $91.3 \pm 1.8 \%$ of $12 \mathrm{~S}$ rRNA and $88.9 \pm 4.9 \%$ of $16 \mathrm{~S}$ rRNA gene sequence reads at a minimum of genus (Table 2). Of 19 total fish taxa detected, including 7 orders and 11 families, 17 were identified to species, with a mean of $15.1 \pm 3.2$ OTUs and $2.3 \pm 0.3$ fishes identified in $12 S$ rRNA fecal samples and $6.8 \pm 2.3$ OTUs and 


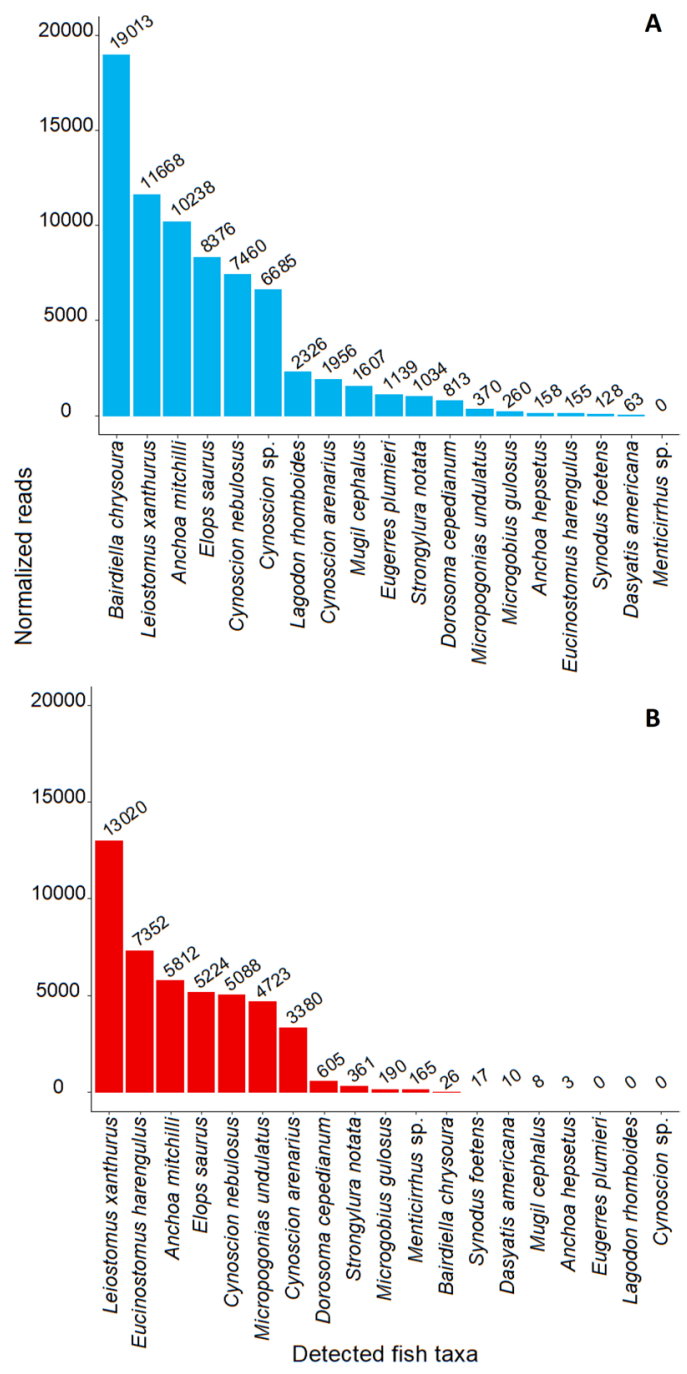

Figure 4. Normalized abundance of detected fish prey taxa in mitochondrial $12 \mathrm{~S}(\mathrm{~A})$ and $16 \mathrm{~S}$ (B) rRNA gene sequence reads from smalltooth sawfish fecal samples. In the 12S rRNA gene analysis, 18 taxa were identified, and in the $16 \mathrm{~S}$ rRNA gene analysis, 16 taxa were identified. The same taxa were included in both graphs for comparative purposes.

$1.7 \pm 0.4$ fishes identified in $16 \mathrm{~S}$ rRNA fecal samples. Silver perch (Bairdiella chrysoura), bay anchovy (Anchoa mitchilli), tidewater mojarra (Eucinostomus harengulus), spotted seatrout (Cynoscion nebulosus), ladyfish (Elops saurus), and spot (Leiostomus xanthurus) were most prevalent in our analysis (Figs. 3 and 4; Supplementary Table S6). For each gene, Bray Curtis similarity indices were calculated after removing individuals in which no prey taxa were detected. A hierarchical clustering based on these results showed that similar river originated samples (i.e., Peace River and Caloosahatchee River) exhibited similarities and clustered together (Supplementary Fig. S1).

The 16S rRNA primer set appeared to be less sensitive than the 12S rRNA primer set when comparing samples sequenced for both genes. In $16 \mathrm{~S}$ rRNA analysis, only three (all tidewater mojarra) of 26 identification instances were not corroborated by the other gene, whereas for $12 \mathrm{~S}$ rRNA, 16 of 34 identification instances were not corroborated. Additionally, the $16 \mathrm{~S}$ rRNA primer set was unable to identify any fish taxa for two samples (SF3, SF12), and striped mojarra (Eugerres plumieri) and pinfish (Lagodon rhomboides) were only identified using the $12 \mathrm{~S}$ rRNA primer set (Fig. 3). While two seatrout species, sand seatrout (C. arenarius) and spotted seatrout (C. nebulosus), were identified in both analyses, species were not always distinguishable in our 12S rRNA analysis with some identifications relegated to Cynoscion sp., indicating that multiple primer sets may be desirable to overcome potential biases.

\section{Discussion}

Traditional dietary studies of fishes have mainly focused on stomach content analysis, using invasive methods that cannot be used to study Critically Endangered species such as the smalltooth sawfish. DNA-based prey identification can overcome many of these limitations, and a variety of molecular methods have been developed over the past decade to enable the investigation of feeding ecology at unprecedented resolution ${ }^{13-16}$. Moreover, even 
heavily digested prey remains can be identified by these methods to track trophic links ${ }^{17}$. Molecular techniques have been used to assess the diet of piscivores with a focus on marine predators such as pinnipeds ${ }^{18}$, squids ${ }^{19}$, and seabirds ${ }^{20}$; however, this is the first study achieving species level resolution for elasmobranchs. In addition, there has been limited application of these techniques for studying the feeding ecology of fishes more generally ${ }^{21}$.

In the present study, we combined multiple molecular markers to identify fish prey to species. Mitochondrial $12 \mathrm{~S}$ and $16 \mathrm{~S}$ rRNA genes were used to target fishes, which were previously identified as the largest fraction of smalltooth sawfish prey taxa via the $18 \mathrm{~S}$ rRNA gene ${ }^{11}$. Using a set of high-resolution mitochondrial genes expanded our results by accounting for differences between the two primer sets, with samples exhibiting variation in taxa detection and proportions between them. An example of the need for multiple genes occurred with silver perch and tidewater mojarra which were often detected together but exhibited drastic differences in sequence proportions between genes. In some samples, the silver perch was only detected by $12 \mathrm{~S}$ rRNA while tidewater mojarra was only detected using $16 \mathrm{~S}$ rRNA. At first, we thought this indicated an error in our analysis or sample preparation for Sanger sequencing, but after comparing identified query sequences of both species for both genes, our sequences of both species, and reference sequences of congeners, all sequences identified as one or the other species via multiple sequence alignment. This process indicated that both sequences belong to these two species, were well differentiated from each other, were similar to other species within their respective genera, and met our threshold for valid identifications. Without the use of two overlapping high taxonomic resolution primer sets, there would have been undetected or undervalued taxa due to biases of individual primer sets and their respective genes.

We also used species-signature sequences consisting of around $40 \mathrm{bp}$ to refine our data, increasing the number of successfully identified sequences and OTUs. However, in two samples, we were unable to differentiate species in the genus Cynoscion for $12 \mathrm{~S}$ rRNA OTUs. The only additional Cynoscion species not identified in the study was the silver seatrout (C. nothus), which is not known to use the estuary where these samples were collected ${ }^{12,22}$. In one sample, we were unable to differentiate species in the genus Menticirrhus for 16S rRNA OTUs. Although our identification protocol identified these as M. littoralis, this species is not known to use the estuary where this sample was collected ${ }^{22}$, and relevant reference sequences of the two possible other Menticirrhus species (i.e., $M$. americanus and $M$. saxatilus) were absent from DNA databases. Further, Pompanon and colleagues ${ }^{14}$ discussed various potential biases of this new technology that could hamper quantitative questions, such as differences in amplification efficiency or DNA survival during digestion. Because the methodology is progressing rapidly, these problems might be minimized in the near future; however, some issues, such as variation in prey size and the time the prey was consumed, are not easily addressed. For example, we were unable to rule out instances of secondary predation (i.e., consumption of a predator which has consumed the target prey), which has not been addressed in any vertebrate predator ${ }^{13,23}$. These challenges are generally present in feeding ecology analyses using traditional techniques and are not limited to molecular methods ${ }^{24}$.

A high-throughput sequencing approach, able to detect trace amounts of prey, was first used on sawfish as a complementary method to assist studies using stable isotopes, a powerful and widely accepted technique in which trophic levels can be identified, but species level prey identification is impossible ${ }^{11,25-29}$. Poulakis and colleagues ${ }^{11}$ studied the trophic ecology of the smalltooth sawfish in South Florida using a combination of stable isotopes and $18 \mathrm{~S}$ rRNA gene sequencing of fecal samples, providing evidence that the species feeds primarily on teleost and elasmobranch fishes. In the present study, we replicated and built on this technique with a larger sample size, resulting in successful identification of more prey and reaffirming the previous conclusion that this species is piscivorous. Furthermore, the prey identified comprised diverse taxa (19 fish taxa from 7 orders and 11 families), indicating that this species is a generalist piscivore. Fish prey items were detected in fecal samples from all individuals with the exception of one, which fed primarily on penaeid shrimp, which we speculate was scavenged discarded bait.

The fish prey we identified mirrored the habitats juvenile smalltooth sawfish are known to use within the Charlotte Harbor estuary ${ }^{30}$. Sawfish preyed on species such as the tidewater mojarra that tends to be found along mangrove shorelines, silver perch and spotted seatrout that are typically found on offshore seagrass flats, and bay anchovies and pinfish that are found in both habitats ${ }^{31}$. Acoustic tracking and monitoring in the study area has shown that smalltooth sawfish use all habitats available to them ${ }^{32,33}$, and the present study suggests that they feed on fishes in all of these habitats. Additionally, we did not observe an ontogenetic dietary shift in our data, which is a trait common amongst elasmobranchs ${ }^{34,35}$. No clear differences in prey were observed between juvenile age classes (less than 1 year old: $<150 \mathrm{~cm} \mathrm{STL}$; greater than 1 year old: $>150 \mathrm{~cm} \mathrm{STL}^{36}$ ), with fishes such as bay anchovy, ladyfish, and silver perch found in each. A dietary shift may become evident if more samples from larger juveniles and adults could be collected as these age classes are known to use more diverse habitats ${ }^{3,11}$.

Although we know little about sawfish feeding behavior and diet beyond anecdotal reports and previous stable isotope analysis ${ }^{11}$, this study presents multiple new findings, such as the presence of anchovies, one of the smallest fish prey items identified, and the southern stingray, which was anticipated by the 18S rRNA analysis that identified Myliobatiformes in the diet ${ }^{11}$. Recent behavioral experiments have shown captive sawfish feeding with rapid lateral swipes of their rostra in the water column and close to the bottom ${ }^{37}$. Our data suggest that smalltooth sawfish are generalist piscivores that feed on pelagic and benthic fishes.

These findings will aid recovery planning for the smalltooth sawfish and the molecular approach used has the potential to reveal previously unknown predator-prey associations from a wide range of taxa, especially protected species. This study shows that smalltooth sawfish consume a variety of fish prey species found across a diverse range of estuarine habitats. Thus, maintaining healthy estuaries, including healthy fish populations, will be important for promoting recovery of the smalltooth sawfish population and should be more effective than species-specific management approaches. Additionally, climate change and associated environmental impacts may destabilize current habitats for the species and alter habitat use, further emphasizing the need for ecosystem level conservation ${ }^{38}$. 


\section{Materials and Methods}

Smalltooth sawfish fecal sample collection and DNA extraction. From 2010 to 2015,16 fecal samples were opportunistically obtained from primarily juvenile smalltooth sawfish in southwest Florida during ongoing field sampling or from necropsies (Table 1). All samples were stored at $-20^{\circ} \mathrm{C}$ until further analysis. DNA extractions were performed using the Quick-DNA Fecal/Soil Microbe Kits (Zymo Research, Irvine, CA, USA) according to manufacturer instructions. DNA was unable to be extracted from one sample (SF2) due to an insufficient amount of feces and 15 fecal samples were used in further sequencing analysis. All methods were carried out in accordance with relevant guidelines and regulations.

High-throughput sequencing. The extracted DNA samples were used for Illumina sequencing to determine the 18S rRNA, mitochondrial $12 \mathrm{~S}$ and $16 \mathrm{~S}$ rRNA genes. Amplification of the 18S rRNA gene was conducted using a universal primer pair (TAReuk454FWD1 [CCA GCA SCY GCG GTA ATT CC] and TAReukREV3 [ACT TTC GTT CTT GAT YRA]). We also applied Illumina sequencing to mitochondrial 12S and 16S rRNA genes to detect fish taxa ${ }^{39}$. The mitochondrial $12 \mathrm{~S}$ rRNA gene was amplified using a universal primer pair for Actinopterygii (Ac12Sf [ACT GGG ATT AGA TAC CCC ACT ATG] and Ac12Sr [GAG AGT GAC GGG CGG TGT]). The mitochondrial 16S rRNA gene was amplified using a universal primer pair for Actinopterygii (Ac16Sf [CCT TTT GCA TCA TGA TTT AGC] and Ac16Sr [CAG GTG GCT GCT TTT AGG C]). The DNA samples were amplified for sequencing in a two-step process. The forward primer was constructed $\left(5^{\prime}-3^{\prime}\right)$ with the forward Illumina overhang adapter (TCG TCG GCA GCG TCA GAT GTG TAT AAG AGA CAG) added to the forward primer used. The reverse primer was constructed $\left(5^{\prime}-3^{\prime}\right)$ with the reverse Illumina overhang adapter (GTC TCG TGG GCT CGG AGA TGT GTA TAA GAG ACA G) added to the reverse primer used. Amplifications were performed in a $25 \mu \mathrm{L}$ reaction with the Qiagen HotStar Taq master mix (Qiagen, Valencia, California, USA), $1 \mu \mathrm{L}$ of each $5 \mu \mathrm{M}$ primer, and $1 \mu \mathrm{L}$ of template. Reactions were performed on the ABI Veriti thermocycler (Applied Biosytems, Carlsbad, California, USA) under the following thermal profile for $18 \mathrm{~S}$ rRNA: an initial denaturation for $5 \mathrm{~min}$ at $95^{\circ} \mathrm{C}$, followed by 35 cycles of denaturation for $30 \mathrm{~s}$ at $94^{\circ} \mathrm{C}$, annealing for $1.5 \mathrm{~min}$ at $50^{\circ} \mathrm{C}$ and ramp up at $0.5^{\circ} \mathrm{C}$ per cycle $(1.5 \mathrm{~min})$ in the first 10 cycles and the following 25 cycles at $54^{\circ} \mathrm{C}$, and extension for $1 \mathrm{~min}$ at $72^{\circ} \mathrm{C}$, with a final extension of $10 \mathrm{~min}$ at $72^{\circ} \mathrm{C}$. In $12 \mathrm{~S}$ and $16 \mathrm{~S}$ rRNA analyses, we used the same thermal profile, but with a constant annealing temperature of $54^{\circ} \mathrm{C}$ for all 35 cycles. The PCR product from the first stage amplification was added to a second PCR based on qualitatively determined concentrations. Primers for the second PCR were designed based on the Illumina Nextera PCR primers as follows: forward - AAT GAT ACG GCG ACC ACC GAG ATC TAC AC [i5index] TCG TCG GCA GCG TC and reverse - CAA GCA GAA GAC GGC ATA CGA GAT [i7index] GTC TCG TGG GCT CGG. The second stage amplification was run the same as the first except with only 10 cycle extensions. For the 18S rRNA gene, the first stage thermal profile used in 12S and 16S rRNA gene amplification was applied for these 10 cycles. Amplicons were visualized with eGels (Life Technologies, Grand Island, New York, USA) and products were pooled equimolar and each pool was size selected in two rounds using SPRIselect reagent (Beckman Coulter, Indianapolis, Indiana, USA) in a 0.75 ratio for both rounds. Size selected pools were then quantified using the Qubit 4 fluorometer (Life Technologies). The final library pool was analyzed on the MiSeq system (Illumina, San Diego, California, USA) using a Miseq reagent kit v3 (Illumina) with pair-end run setting of $2 \times 300$ flow cell at $10 \mathrm{pM}$. We ran $12 \mathrm{~S}$ and $16 \mathrm{~S}$ rRNA gene amplification on the same Miseq reagent kit, and $18 \mathrm{~S}$ rRNA gene was run separately. All PCR reactions were run with no-template control; the negative control was also tagged and sequenced along with samples for contamination check purposes. Illumina sequencing was performed at RTL Genomics (Lubbock, Texas, USA).

To analyze the sequence data, forward and reverse reads were taken in FASTQ format and merged using PEAR Illumina paired-end read merger ${ }^{40}$. The formatted FASTQ files were then converted into FASTA-formatted files for subsequent analyses. DNA sequences were clustered into OTUs at 97\% similarity using the CD-Hit-Est clustering algorithm ${ }^{41,42}$. OTUs consisting of less than five sequences were considered inconsequential and omitted from further analyses (12S rRNA gene sequences omitted: $2.06 \pm 0.23 \%$ [mean \pm SE]; $16 \mathrm{~S}$ rRNA: $0.65 \pm 0.08 \%$; $18 \mathrm{~S}$ rRNA: $1.28 \pm 0.06 \%$ ), with the remainder entering our identification protocol using BLAST.

Sequencing error of $12 \mathrm{~S}$ and $16 \mathrm{~S}$ rRNA genes was estimated to be $2 \%$ by comparing OTU centroid sequences identified as smalltooth sawfish by BLAST and their respective similarity percentages with a reference sequence (GenBank Accession: KP400584) for each gene via multiple sequence alignment using the MUSCLE program within MEGA7 software ${ }^{43}$ to manually check for chimeric sequences (Supplementary Fig. S2). This estimated sequencing error was used in our sequence identification protocol. Sequencing error was not estimated for $18 \mathrm{~S}$ rRNA gene analysis due to its low associated taxonomic resolution, with the taxonomic class of the highest scoring BLAST similarity result accepted for each OTU. When class was not applicable, the next highest taxonomic level was used. Taxonomy follows Page and colleagues ${ }^{44}$ for fishes and Williams ${ }^{45}$ for crustaceans.

Mitochondrial 12S and 16S rRNA gene sequence identification protocol. Based on our estimation of sequencing error found in the host, BLAST results with a similarity score $\geq 98 \%$ were accepted for each prey OTU. If this criterion was met and ties were present, OTUs would be moved to the next step of our identification protocol. The remaining OTUs along with those with ties were aligned with $\sim 40 \mathrm{bp}$ species signature sequences identified for all fish species documented in the sample collection area ${ }^{12}$ that either had reference sequences available in GenBank or were sequenced for this study (Supplementary Table S1). This tag-sequencing strategy determined $\sim 40$ bp species signature sequences based on available data, with each sequence checked for specificity via BLAST. Exact matches were accepted as an accurate identification. If an exact match was not made with a tied OTU, the lowest shared taxonomic classification between the tied BLAST results was accepted (none were found higher than genus level, and all were Cynoscion sp.). All results were checked against available fisheries data $^{22}$, with any dubious identifications relegated to the lowest viable taxonomic classification (none higher than genus level and all were Menticirrhus sp.). 
Sanger sequencing for mitochondrial $12 \mathrm{~S}$ and 16S rRNA genes of potential fish prey. Twenty-four fish species known from our sampling area were sequenced (Fig. 1; Supplementary Table S3). Sixteen species were sequenced for the mitochondrial $12 \mathrm{~S}$ rRNA gene and 23 species for the mitochondrial $16 \mathrm{~S}$ rRNA gene using the same primer sets used in the high-throughput sequencing ${ }^{39}$ except for a minor modification in Ac16Sr(C-) [CAG GTG GCT GCT TTT AGG C]). Preparation of sequencing samples was carried out as described previously ${ }^{11}$.

Data deposit. High-throughput mitochondrial 12S and 16S rRNA, and 18S rRNA gene sequences of smalltooth sawfish fecal samples were deposited in the National Center for Biotechnology Information sequence read archive under accession number SAMN10130720. Mitochondrial 12S and 16S rRNA gene fish sequences were deposited in GenBank under accession numbers MH715297-312 and MH715980-6002, respectively.

Received: 28 July 2019; Accepted: 17 October 2019;

Published online: 04 December 2019

\section{References}

1. Carlson, J., Wiley, T. \& Smith, K. Pristis pectinata. The IUCN Red List of Threatened Species 2013: e.T18175A141791261, https://doi. org/10.2305/IUCN.UK.2013-1.RLTS.T18175A141791261.en (accessed 5 August 2019) (2013).

2. Wueringer, B. E., Squire, L. \& Collin, S. P. The biology of extinct and extant sawfish (Batoidea: Sclerorhynchidae and Pristidae). Rev. Fish Biol. Fish. 19, 445-464 (2009).

3. Brame, A. B. et al. Biology, ecology, and status of the smalltooth sawfish Pristis pectinata in the USA. Endang. Species Res. 39, 9-23 (2019).

4. Blewett, D. A., Hensley, R. A. \& Stevens, P. W. Feeding habits of common snook, Centropomus undecimalis, in Charlotte Harbor, Florida. Gulf. Caribb. Res. 18, 1-14 (2015).

5. Bangley, C. W., Rulifson, R. A. \& Overton, A. S. Evaluating the Efficiency of flushed stomach-tube lavage for collecting stomach contents from dogfish sharks. Southeast. Nat. 12, 523-533 (2013).

6. Dias, E., Morais, P., Leopold, M., Campos, J. \& Antunes, C. Natural born indicators: Great cormorant Phalacrocorax carbo (Aves: Phalacrocoracidae) as monitors of river discharge influence on estuarine ichthyofauna. J. Sea Res. 73, 101-108 (2012).

7. Sinclair, E. H. \& Zeppelin, T. K. Seasonal and spatial differences in diet in the western stock of steller sea lions (Eumetopias Jubatus). J. Mammal. 83, 973-990 (2005).

8. Gagliardi, A., Martinoli, A., Preatoni, D., Wauters, L. A. \& Tosi, G. From mass of body elements to fish biomass: A direct method to quantify food intake of fish eating birds. Hydrobiologia. 583, 213-222 (2007).

9. Mariano-jelicich, R. \& Favero, M. Assessing the diet of the black skimmer through different methodologies: is the analysis of pellets reliable? Waterbirds. 29, 81-87 (2006).

10. Barrett, R. T. Food web interactions in the southwestern Barents Sea: Black-legged kittiwakes Rissa tridactyla respond negatively to an increase in herring Clupea harengus. Mar. Ecol. Prog. Ser. 349, 269-276 (2007).

11. Poulakis, G. R. et al. Sympatric elasmobranchs and fecal samples provide insight into the trophic ecology of the smalltooth sawfish. Endang. Species Res. 32, 491-506 (2017).

12. Poulakis, G. R., Matheson, R. E. Jr., Mitchell, M. E., Blewett, D. A. \& Idelberger, C. F. Fishes of the Charlotte Harbor estuarine system, Florida. Gulf Mex. Sci. 2, 117-150 (2004).

13. King, R. A., Read, D. S., Traugott, M. \& Symondson, W. O. C. Molecular analysis of predation: A review of best practice for DNAbased approaches. Mol. Ecol. 17, 947-963 (2008).

14. Pompanon, F. et al. Who is eating what: Diet assessment using next generation sequencing. Mol. Ecol. 21, 1931-1950 (2012).

15. Symondson, W. O. C. \& Harwood, J. D. Special issue on molecular detection of trophic interactions: Unpicking the tangled bank. Mol. Ecol. 23, 947-693 (2014).

16. Traugott, M., Kamenova, S., Ruess, L., Seeber, J. \& Plantegenest, M. Empirically characterising trophic networks: what emerging dna-based methods, stable isotope and fatty acid analyses can offer. Adv. Ecol. Res. 49, 177-224 (2013).

17. Hindell, M. A., Gales, N. J., Jarman, S. N., Casper, R. M. \& Deagle, B. E. Detecting prey from DNA in predator scats: A comparison with morphological analysis, using Arctocephalus seals fed a known diet. J. Exp. Mar. Bio. Ecol. 347, 144-154 (2007).

18. Deagle, B. E. et al. Analysis of Australian fur seal diet by pyrosequencing prey DNA in faeces. Mol. Ecol. 18, 2022-2038 (2009).

19. Deagle, B. E. et al. Molecular scatology as a tool to study diet: Analysis of prey DNA in scats from captive Steller sea lions. Mol. Ecol. 14, 1831-1842 (2005).

20. Bowser, A. K., Diamond, A. W. \& Addison, J. A. From puffins to plankton: A DNA-based analysis of a seabird food chain in the northern Gulf of Maine. PLoS One 8, 1-17 (2013).

21. Leray, M., Meyer, C. P. \& Mills, S. C. Metabarcoding dietary analysis of coral dwelling predatory fish demonstrates the minor contribution of coral mutualists to their highly partitioned, generalist diet. PeerJ 3, e1047 (2015).

22. Idelberger, C. F. \& Greenwood, M. F. D. Seasonal variation in fish assemblages within the estuarine portions of the Myakka and Peace Rivers, southwest Florida. Gulf Mex. Sci. 23, 224-240 (2005).

23. Hardy, N. et al. Assessing the trophic ecology of top predators across a recolonisation frontier using DNA metabarcoding of diets. Mar. Ecol. Prog. Ser. 573, 237-254 (2017).

24. Johnson, J. H., DiRado, J. A., Mackey, G. \& Abbet, R. Comparative diets and foraging strategies of subyearling Atlantic salmon, brown trout, and rainbow trout during winter. J. Appl. Ichthyol. 33, 1158-1165 (2017).

25. Olin, J. A. et al. Variable $\delta^{15} \mathrm{~N}$ diet-tissue discrimination factors among sharks: implications for trophic position, diet and food web models. PLoS One 8, 1-12 (2013).

26. Hussey, N. E., Chapman, D. D., Donnelly, E., Abercrombie, D. L. \& Fisk, A. T. Fin-icky samples: An assessment of shark fin as a source material for stable isotope analysis. Limnol. Oceanogr. Methods. 9, 524-532 (2011).

27. Hussey, N. E. et al. Stable isotopes and elasmobranchs: tissue types, methods, applications and assumptions. J. Fish Biol. 80, 1449-1484 (2012).

28. Hussey, N. E. et al. Size-based analysis of diet and trophic position of the white shark Carcharodon carcharias in South African waters in global perspectives on the biology and life history of the white shark, M. Domeier, Eds, pp. 27-48 (CRC Press, 2012).

29. Malpica-Cruz, L., Herzka, S. Z., Sosa-Nishizaki, O. \& Lazo, J. P. Tissue-specific isotope trophic discrimination factors and turnover rates in a marine elasmobranch: empirical and modeling results. Can. J. Fish. Aquat. Sci. 69, 551-564 (2012).

30. Poulakis, G. R., Stevens, P. W., Timmers, A. A., Wiley, T. R. \& Simpfendorfer, C. A. Abiotic affinities and spatiotemporal distribution of the endangered smalltooth sawfish, Pristis pectinata, in a south-western Florida nursery. Mar. Freshw. Res. 62, 1165-1177 (2011).

31. Poulakis, G. R., Blewett, D. A. \& Mitchell, M. E. The effects of season and proximity to fringing mangroves on seagrass-associated fish communities in Charlotte Harbor, Florida. Gulf Mex. Sci. 21, 171-184 (2003).

32. Poulakis, G. R., Stevens, P. W., Timmers, A. A., Stafford, C. J. \& Simpfendorfer, C. A. Movements of juvenile endangered smalltooth sawfish, Pristis pectinata, in an estuarine river system: Use of non-main-stem river habitats and lagged responses to freshwater inflow-related changes. Environ. Biol. Fishes. 96, 763-778 (2013). 
33. Huston, C. A. et al. Diel movements of juvenile smalltooth sawfish: Implications for defining the size of a nursery hotspot. Endang. Species Res. 34, 311-322 (2017).

34. Bethea, D. M., Buckel, J. A. \& Carlson, J. K. Foraging ecology of the early life stages of four sympatric shark species. Mar. Ecol. Prog. Ser. 268, 245-264 (2004).

35. Newman, S. P., Handy, R. D. \& Gruber, S. H. Ontogenetic diet shifts and prey selection in nursery bound lemon sharks, Negaprion brevirostris, indicate a flexible foraging tactic. Environ. Biol. Fishes. 95, 115-126 (2012).

36. Scharer, R. M., Patterson, W. F., Carlson, J. K. \& Poulakis, G. R. Age and growth of endangered smalltooth sawfish (Pristis pectinata) verified with LA-ICP-MS analysis of vertebrae. PLoS One 7, e47850 (2012).

37. Wueringer, B. E., Squire, L., Kajiura, S. M., Hart, N. S. \& Collin, S. P. The function of the sawfish's saw. Curr. Biol. 22, R150-R151 (2012).

38. Poulakis, G. R. \& Grubbs, R. D. Biology and ecology of sawfishes: global status of research and future outlook. Endang. Species Res. 39, 77-90 (2019).

39. Evans, N. T. et al. Quantification of mesocosm fish and amphibian species diversity via environmental DNA metabarcoding. Mol. Ecol. Resour. 16, 29-41 (2016).

40. Zhang, J., Kobert, K., Flouri, T. \& Stamatakis, A. PEAR: a fast and accurate Illumina paired-end read merger. Bioinformatics 30, 614-620 (2014)

41. Huang, Y., Niu, B., Gao, Y., Fu, L. \& Li, W. CD-HIT suite: A web server for clustering and comparing biological sequences. Bioinformatics 26, 680-682 (2010).

42. Li, W. \& Godzik, A. Cd-hit: A fast program for clustering and comparing large sets of protein or nucleotide sequences. Bioinformatics 22, 1658-1659 (2006).

43. Kumar, S., Stecher, G. \& Tamura, K. MEGA7: molecular evolutionary genetics analysis version 7.0 for bigger datasets. Mol. Biol. Evol. 33, 1870-1874 (2016).

44. Page, L. et al. Common and scientific names of fishes from the United States, Canada, and Mexico. $7^{\text {th }} \mathrm{ed}$. (American Fisheries Society, Bethesda, 2013).

45. Williams, A. Shrimps, lobsters, and crabs of the Atlantic coast of the eastern United States, Maine to Florida (Smithsonian Institution, Washington, D.C., 1984).

\section{Acknowledgements}

Our smalltooth sawfish research is ongoing and has been supported primarily by funding from the U.S. Department of Commerce (DOC), National Oceanic and Atmospheric Administration's (NOAA) National Marine Fisheries Service through Section 6 (Cooperation with the States) of the U.S. Endangered Species Act under the following grant awards to the Florida Fish and Wildlife Conservation Commission from NOAA: NA10NMF4720032 and NA13NMF4720047. Part of this research was also supported by the Save Our Seas Foundation, Marco Island Shell Club, the Florida Gulf Coast University Whitaker Center for STEM Education, and the Blair Foundation. Statements, findings, conclusions, and recommendations are those of the authors and do not necessarily reflect the views or policies of the funders. This research was conducted under Endangered Species Permit numbers 1475 and 15802 issued to GRP by NOAA Fisheries.

\section{Author contributions}

T.L.H. and H.U. conducted laboratory analyses, G.R.P. and R.M.S. conducted sample collection, and all authors (T.L.H., H.U., G.R.P., R.M.S. and S.G.T.) contributed to data interpretation and manuscript writing.

\section{Competing interests}

The authors declare no competing interests.

\section{Additional information}

Supplementary information is available for this paper at https://doi.org/10.1038/s41598-019-53931-7.

Correspondence and requests for materials should be addressed to H.U.

Reprints and permissions information is available at www.nature.com/reprints.

Publisher's note Springer Nature remains neutral with regard to jurisdictional claims in published maps and institutional affiliations.

Open Access This article is licensed under a Creative Commons Attribution 4.0 International License, which permits use, sharing, adaptation, distribution and reproduction in any medium or format, as long as you give appropriate credit to the original author(s) and the source, provide a link to the Creative Commons license, and indicate if changes were made. The images or other third party material in this article are included in the article's Creative Commons license, unless indicated otherwise in a credit line to the material. If material is not included in the article's Creative Commons license and your intended use is not permitted by statutory regulation or exceeds the permitted use, you will need to obtain permission directly from the copyright holder. To view a copy of this license, visit http://creativecommons.org/licenses/by/4.0/.

(C) The Author(s) 2019 